\title{
Connected Platforms for the Non-typical User: Design Methodology to Observe the Outliers
}

\author{
Inés Alvarez-Icaza ${ }^{1 *}$, Arturo Molina ${ }^{2}$, Rogelio Bustamante-Bello ${ }^{2}$ \\ 1 Tecnologico de Monterrey, School of Architecture, Art and Design, Design Department, Del Puente \#222, \\ 14318, Mexico City, Mexico. \\ 2 Tecnologico de Monterrey, School of Science and Engineering, Mexico City, Mexico. \\ * Corresponding author. Tel.: +525533289344; email: i.alvarezicaza@tec.mx \\ Manuscript submitted December 10, 2019; accepted March 5, 2020.
}

doi: 10.17706/ijcce.2020.9.3.122-133

\begin{abstract}
The Smart, Sensing and Sustainable Systems paradigm, known as the "S3 concept", aims to promote the evolution of companies and academic environments through shared and adaptable knowledge. Therefore, this advancement follows new integrated products and services systems (PSS) designed to integrate everyone, everywhere, including non-typical users, i.e. people with disabilities, indigenous communities, elders, etc. By considering this it may facilitate Product and Service Systems design methodologies so to cover the articulate and non-articulate customer needs and in addition, using a social perspective. This research proposes a design framework to consider the non-typical human input analysis, along with the knowledge-sharing models exploration as academic collaboration platforms, to help Design and engineering students in identifying the best possible way of designing inclusive Sensible, Smart and Sustainable Systems, including a Social dimension.
\end{abstract}

Key words: Design methodology, collaborative networks, collaboration platforms, smart products, non-typical user, educational innovation, higher education.

\section{Introduction}

Smart connected products hold at least four types of capabilities: monitoring, control, optimization and autonomy [1]. Thus, products collect information by sensing the environment input, which may come from a system or from a user. When products record data, they send it to a service platform allowing to keep a close relation to "systems that link an array of product systems together" [1]. This document explores the described capabilities used to enhance inclusion in people that normally struggle when using technology products or systems. This occurs when the user's needs are not well attended.

There are different types of user needs and behaviour, related to products features and expressed as product-user communication. Non-articulated needs, described as Natural Society Behaviour [2], are recovered data from a product when the product it is performing its role. During this process, systems collect information about the user to improve the device's features and performance. On the other hand, Non-Natural Behaviour are articulated needs relate to the user's behaviour. In this case, there is an adjusting one's behaviour will for the best performance of the product or the product society. However, some consumers may not be able to adjust their habits and actions, whether they want it or not. During the process of trying, valuable data to scale the product's performance could be gathered and analysed to understand the exact shape and size of the barrier this product might produce. 
The first section of this document provides a theoretical framework and this research contribution. Next, the second segment gives a collaborative models overview and the possible user-device interactions. Finally, the third section exposes the previous work that lead, in the final part of this document, to the framework definition applied to a collaborative design methodology for Inclusive Smart, Sensing, Sustainable and Social $\left(\mathrm{S}^{4}\right)$ products and service systems [3]. Do not change the font sizes or line spacing to squeeze more text into a limited number of pages. Use italics for emphasis; do not underline.

\section{The Open Innovation Paradigm}

Open Innovation, described by Chesbrough [4] as a description and complex processes exploration resource, originated by reciprocal interaction pursuing collaboration, between people, organizations and technologies [5]. This collaborative interaction may take place in external entities, such as clients, suppliers, experts, universities, Research and Development private or public institutions, partners, competitors, and communities altogether [6].

This concept has certainly addressed this problem, and to be fair, in an efficient and democratically way shared knowledge for everyone. However, with a single-road impact: decision-makers need to trust simulations to use their results as decision support. Though, difficult and complex decisions, coming from embracing real-world challenges, require careful review through all possibilities [7]. Considering imaginable variations of a desirable outcome through the design process, ought to hold a collaborative and interconnected way between people, devices and knowledge. Therefore, we are facing "a paradigm shift in innovation, where the industrial thinking becomes obsolete and makes room for new post-industrial in which the focus is moved from pure economic growth to a more holistic approach to human well-being" [8].

Open Innovation intermediaries exist to stimulate interaction and networking among stakeholders of the innovation process [6]. Through digital complex platforms, these intermediaries provide a service for individuals and organizations to "cross-fertilize their resources and competences, in a wide variety of specialized fields, providing a wide range of services". If the collected outlier -or non-typical users- data are identified and analysed to share and compare knowledge from diverse contexts in the world within academic environments, it could speed up innovation and inclusion processes.

Open Innovation will play a key role in the developed economies over the next decade; we can previsualize technological trends that will fuel innovation (from blockchain to digitalization) strengthening the shift for more and better cooperation, while enhancing the social and sustainable development dimension.

Currently, Open Innovation to takes place in many ways: Makerspaces, Fab-Labs, High Tech-Labs, Creativity Rooms, Living Labs, among others [9]. They represent the following innovation era after Research and Development Departments in companies [10]. Open Innovation Labs (OILab) are one remarkable shape that brings together the benefits of this perspective in physical and tangible environments, during the entire innovation process. Multiple institutions, organizations, and even, companies include OILabs as an innovation strategy to promote and support interactive internal and external collaboration (i.e. students from a university with a company innovation team). This research proposes using OILabs concept in collaborative platforms with information at reach, for inclusion purposes when looking for innovation opportunities. Understanding different ways to collaborate exist, next section explores some collaborative models and purposes, to set a suitable combination for the pursued objective in this work.

\section{Collaborative Models}

Most typical technology users -in the shape of smartphones, computers, apps or other complex gadgets; are adapted to the Information and Communication Technology (ICT) world. On the other hand, non-typical 
users: elders, people with disabilities, young children, indigenous people, etc. when facing a technological challenge, usually they thrive through time, by proof and error. However, in the process of trying valuable information gets lost. This human input, on a global scale, could be the source of better smart and self-adaptable systems, demanding a high-level of automation [11]. At his level, systems understand and record the user's needs and to make decisions according to the system and users pursued performance. This research explores the possibility of reframing the problem of what $\mathrm{S}^{3}$ products designers and developer ought to consider when looking for fostering and speeding up innovation.

From the strategic design perspective, it is adequate to consider that socio-technological innovation has two sides of a coin: the technical dimension (production, distribution and consumption) and the social dimension (relations, behaviour and culture), both relevant and interdependent. This innovation type gathers technical skills, proper technology and specialized knowledge in a local level, combined with complementary external experience, along with needs, aspirations, life contexts, history and community traditional information [8]. Understanding the potential combinations of these two socio-technical innovation aspects, means an emerging need for managing the phenomena observation in which the combination occurs; this situation unavoidably leads to collaboration.

Because connectivity, the complex design processes are becoming co-design processes [9], as an outcome, the need of setting a common language and collaborative environments conditions, emerge as an irrevocable demand. While Industry 4.0 represents a redesign opportunity, moving towards evolved and smart products and service systems, it is convenient to find a way to feed it, through highlighting the variety of reality interpretation ways, in different context around the globe. If social responsibility is an obligation for those who care about equality, then it is also the diversity acknowledgement, along with the emerging needs and global inter-contextual requirements.

In the quest for innovation, "organizations need to develop places where they can come together and work creatively-that is, platforms for collaboration" [10], Furthermore, a main driver of this transformation towards collaboration are the social networks invention and smart devices, combined with the employees' and users' appealing to them. These enablers are the technological and organizational foundation to, eventually, release collaboration productivity as the major productivity growth source in Industry 4.0 [7].

Both, public and private research entities are aware of the importance of access to interconnected data as an intellectual knowledge source for growth and effectiveness in the innovation process [11]. This idea can lead to a scenario in which collaboration, co-design processes and co-creation of new PSS, could be a significant shift in the way we ideate improvements and innovation on global level. Thus, this could be sheltered in a variety of means, in which actors may be internal or external to companies and organizations. Experimentation platforms would shape as a trial environment providing "a neutral environment for building and testing solutions in simulated or 'near real-world' contexts" [12].

To reach the product technologies complexity and manufacturing techniques means physical components integration and Intelligent Technological systems for developing and applying cyber-physical technical systems in product manufacturing and global service scenarios [12]. Within the Internet of Things, in the cyber-physical environment, it can occur three communication variations: human-human, human-machine, and machine-machine [13]; this interactions, eventually can take place to raise collaboration.

Furthermore, it is relevant to observe that there are several motivations to collaborate and communicate, and many collaborative models [14], each one with a distinctive role and stakeholder's combination, which can be grouped as follows:

a) Collaborative Networked Organizations (CNO) are described as a group of independent organizations collaborating to achieve common or compatibles goals, and whose interactions are supported by computer networks. [15] 
b) Customer Communities (CC), which exist to "engage customers in an on-going dialogue with other customers and also with the firm". These communities allow customers to take several roles, such as Co-designers, Innovators, Socially Responsible Actors or even Lead-Users, to name a few.

c) Experience-centric Networks (ECN), which depend on the device's interface as network nodes, connected to e-platforms, collecting the user experience input, using the communication flow for firm-customer interaction. This scenario allows the user to determine personal experiences in a fast, simple and secure way.

The list above, describe the three most relevant ways of operating collaboration in an interconnected environment. These three interconnected system operating ways will provide better, faster, more comfortable and more adequate devices, and the interactions beneficiaries are the collaboration stakeholders. In the first case, the $\mathrm{CNO}$, large organizations pursuing one common goal will receive information to reach or to get close to that goal. In the second case, a company, fostering customer's ideas and product or service engagement, runs the CC. Finally, the ECN is focused on the experience of using interconnected devices to improve or innovate that experience.

Today, the three collaboration models operators: Companies, Users and service-product system designers operate independently and with their own language: there is a great barrier, physical, digital and about information, which prevents spontaneous and organized collaboration. There is a fourth option: to operate collaboration for someone who is still-not the user, by an organization who is not the owner or the product-service system provider but using everyone's digital environment. This combination usually takes place in academic environments. The operators' purposes will go then, from the creation and management of the marketplace to the technological innovations exchange, sometimes providing services for the production process or scouting new ideas [13].

Consequently, there are also three plausible collaboration outcomes, depending on the collaboration objective type and approach: for the exploration type, with the objective of defining core problems or connect with problem solvers, the outcome could be a "Shared definition of the problem or a list of potential solutions". When pursuing developing solution or testing prototypes in near- real-world contexts -the experimentation type-, the outcome will be an "Assessments of possible solutions or Solution recommendations". In the third type, which relates to Execution i.e. building and disseminating solution templates or helping adopters adapt to system-wide changes, the expected outcome should be a "Solution templates, Implementation standards or a Rapid adoption of the social innovation" [13].

Therefore, while this research desired outcome is experimentation, the communication type should be between the Device and the User to extract as much interactive information as possible. Academic research interdisciplinary teams will operate a Collaboration platform, to provide a solution network for the integration and inclusion for non-typical users. Table 1 shows this combination as Collaboration Type 2: Human-Device; in Experience Centric Networks, operated by the designer/developer; for experimentation purposes.

Table 1. Communication and Collaboration Types

\begin{tabular}{|l|c|c|c|}
\hline Arrangement criteria & Type 1 & Type 2 & Type 3 \\
\hline By communication type & Human-Human & Human-Device & Machine- Machine \\
\hline By motivation & Customer Communities (CC) & Experience- centric Networks (ECN) & $\begin{array}{c}\text { Collaborative Networked } \\
\text { Org. (CNO) }\end{array}$ \\
\hline By operator & User & Designer/ Developer & Companies \\
\hline By the desired outcome & Exploration & Experimentation & Execution \\
\hline
\end{tabular}




\section{Methodology}

\subsection{Previous Work}

As mentioned in the introduction, this research objective is to identify the best possible way of designing inclusive Sensible, Smart, Sustainable and Social Product-Service Systems, through the outlier's analysis: the non-typical user activity and performance.

To establish this research methodology stages, two previous cases will be presented, used as a starting point to set the process and stages to follow in this work. Each case helped to determine the first stages of the Action Research protocol that will follow in this research:

\subsubsection{Emotional product validation}

A Company who needed to validate a hypothesis set this project's objective. They held that fabric detergent packages could change during some specific seasons of the year, and that consumers would find it appealing. For this matter, 9th-semester design students defined a participatory design process applied to a consumers group from a specific niche.

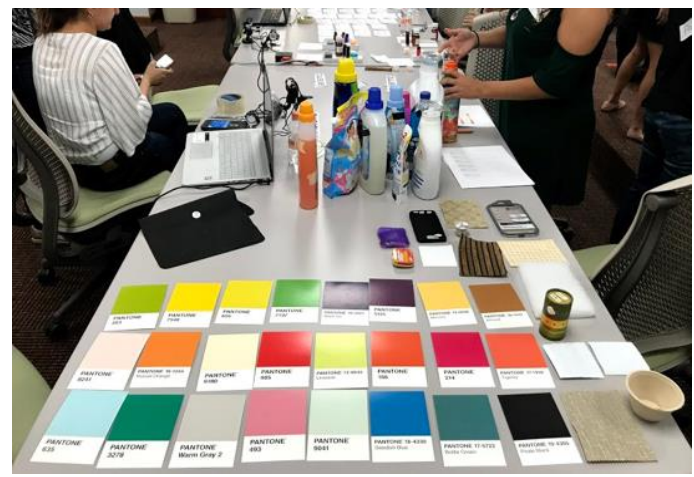

Fig. 1. Participatory activity to define the stimuli set.

The goal was to design the stimuli for the micro-expression measurement. This would provide the first customer's impressions about the idea of having certain variations according to the seasons. During the process, students collected information to generate insights related to colour, shape, odour and sensations expressed by the consumers (Fig. 1); that was the starting point to prove the hypothesis.

For the second stage, the system recorded the consumer's micro expressions when interacting with the packaging 3D printed models, along with the colour-odour combination and the product description. This took place inside the Emotional Domotic Cabin [16], micro-expressions were measure with a galvanic response equipment.

From that process, it was possible to reach a conclusion about the first statement: The perception, as a consumer's first response, was positive regarding the idea of having such stimuli combination in the product package. From this conclusion, the Company was certain about the idea value and decided to start the product and branding development. This project took place between April 2018 and February 2019.

The importance of this case study for the present research is the implementation protocol, which followed a Participative Action Research (PAR) method.

\subsubsection{Validation heuristic model for devices promoting social interaction and physical activity in elderly people}

This research's involvement with the project called "Framework for promoting social interaction and physical activity in elderly people using gamification and fuzzy logic strategy" is limited to the validation strategies for the device interface design, using Nielsen's Heuristic Validation [17] as a guideline. This 
doctoral research project aims to allow a smart and sensible system to learn and adjust to the user's behaviour. The final goal is shaping its performance, reducing energy waste and promoting social engagement for the user, in this case, a people over 65 years old [18].

To reach an adequate design for elders, which would avoid usability problems with the interface elements, the information tools should have specific characteristics. It is convenient to have elements in visible size, thick and clear text and background and front shape contrast in the screen. Also, due to the dexterity and manoeuvrability of elderly people, it is appropriate to set the possibility of reducing the system speed and instructions [19].

Hence, the opportuneness of setting a validation tool, using a systematic process for determining the right interface design. The current stage is the validation through a Heuristic Analysis, along with some specific considerations, such as personality types, linked to the user profile (people over 65 years old).

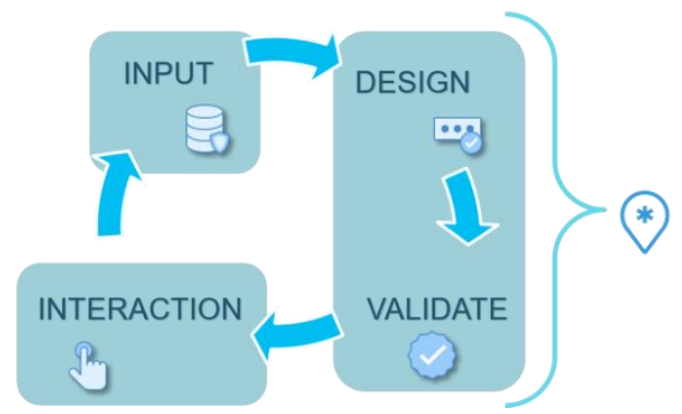

Fig. 2. Use and experience record Loop.

From the interphase proposals heuristic analysis review, focusing in error prevention aspect, information about the possible outcome will enable previsualization of the possible process systematization: a validation strategy for the initial design stage. The identified elements in this process will be used as starting point in the stages to come (see Cycle one section below).

As it has been stated before, non-typical users struggle when using technological devices. Therefore, the proposed validation tool would allow identifying the items prone to cause confusion in the user. By selecting those elements in the design process, it could be possible to include the feature of recording specific interactions outcomes. From the collected data, designers and developers could iterate faster, leading to the system improvement and innovation.

\subsection{Design Process and Subject of Study}

The previous cases have shown the two first research methodology stages for this project. However, it is required to develop a third moment to understand the performance loop (from design to data gathering, see Fig. 2), in which the design process allows to identify those aspects challenging the user's capabilities.

As shown in Fig. 3, the loop is a continuum that could start in the identification of the challenging features, using systematic validation strategy applicable by design and engineering students. As the product reaches the market, the interactive machine learner in the device records the human through interaction with the device: that is the input for the next design iteration.

This research will observe the process between the design and the validation stages ${ }^{1}$ and, the non-typical users: elderly population (60 years old and over). The selection of this age range due to the increasing number of elders in the years to come in overpopulated countries. According to World Health Organization, by the year 2050 will nearly double from $12 \%$ to $22 \%$ [16].

1 This will occur from May 2020 to December 2021. 
As it has been established, the methodology to be followed is action research [17]-[19] with design and engineering students, to systematize the teaching/ learning process and practice and, at the same time, contributing to theoretical knowledge [17]. Although, applying this method requires not only using theory and using it in practice, but "also it designates ongoing and evolving action as part of that process." [19]. This means to involve a series of actions and stages to develop a situation for the "Use and experience record Loop" (Fig. 3) observation, measurement and comparison. This will allow extracting from it, critical elements to use it as a study object to be used in student's design proposals.

$S^{3}$ PSS require specific design principles to achieve standardization customization, personalization, upgrades, and predictive, enhanced, or remote services [1]. Hence, the need of determining the specific task or tasks leading to an inclusive design, that will be observed in this research. Between validation and design stages, depending on the design method, there are a number of actions leading to the result, which in this case is a PSS. To frame a design solution [20], design students evaluate the best way to meet users and clients most valuable design alternative. To highlight the more useful steps and actions for this research's purpose, the observable design process steps, will be presented in order to argue the selected study object for this work:

a) Opportunities identification: The design process can start before this stage, if using methodologies that include designing while researching, however the proper design definition stage is consequence of the opportunity identification. When a need, a problem or a possibility is latent, a design opportunity materializes. It is not evident when nobody is looking for it, but it can appear without preface, just by the events conjunction that may occur in the process of seeking innovation. Usually, this moment's outcome is the design brief, which is to be defined between the designer and the client; however, when the user is involved, the opportunity of using participatory techniques emerges.

b) Design Brief definition: This is the list of requirements, taken from the client or the user, to start designing and determining the features, materials, and technology along with the design concept to be included in the result. Normally, a design brief is an agreement within the design team, about what should be pursued in the design process. This stage's result will be a number of alternatives to combine the requested features and the innovation opportunities. This stage might be called Concept design and target specification.

c) Design Proposal: This is the first response to the brief, the product/system ideation; usually it is delivered to the client or the design team, and analysed under the light of innovation depending on the selected value for the design team (for instance, when using Design Thinking as a design approach or method, it should be Human Desirability, Technical Feasibility and Business Viability [21]). This proposal is an idea to be elaborated in the sub sequential moments of the design process.

d) Alternatives production: An ongoing stage of iterations in which the designer attempts to include innovative features and all the requirements included in the brief. In each iteration, some item or feature should evolve from the previous one. Each alternative can highlight a specific feature and as a conclusion, the design team can determine the most convenient one. A number of possible techniques could be used in this moment: from conveying options to envisioning scenarios, at the end of this process, all the alternatives should be gathered and organized to set a clear hierarchy structure. Ideally, this would lead to the best possible result: the design proposal.

e) Design Solution: This is the last iteration of the cycle; it provides a detailed design that should be put into a validation process with the client/user or the design team. The final design solution selection depends on the design requirements fulfilment the in the most innovative and convenient way. In 
this moment, the idea has a description and a shape in real world, and it can be tested to gather as much information as possible, before the product/system market launching. For systems it also may include an operation plan blueprint and customer journey map.

\subsection{Design methodology for inclusive sensible, smart, sustainable and social PSS, using action research}

From the presented definitions and for this research purpose, the most convenient stage to work with, in this research is the Opportunities Identification stage. Hence, a variety of possibilities to set the best way to reframe the problem of inclusion in smart and connected products and systems.

Observing the possibilities and the design considerations could lead to select the most PSS challenging features for the non-typical user, which as defined before, lacks native abilities to use technology. Is important to mention that although the $\mathrm{S}^{3}$ paradigm was described before, for this research purpose, it is convenient to include a fourth characteristic: the social dimension of devices. This means that devices are connected to each other for monitoring but also for sharing information purposes. In addition, the social dimension means acknowledging that the Product and Service System capabilities must be coherent with non-typical users' equal inclusion. Through this, it is possible "enhancing the global performance of the social structure, that is made to reach the consumer's speciation regarding the product." [2]

As mentioned before, action research sets a commitment to present a "set of research approaches, which includes phenomenological, ethnomethodological and biographical approaches". In consequence, researchers may explore, explain or describe a specific practice through collecting qualitative data to identify key elements, particular insights or recommendations for future practice: a design methodology [19].

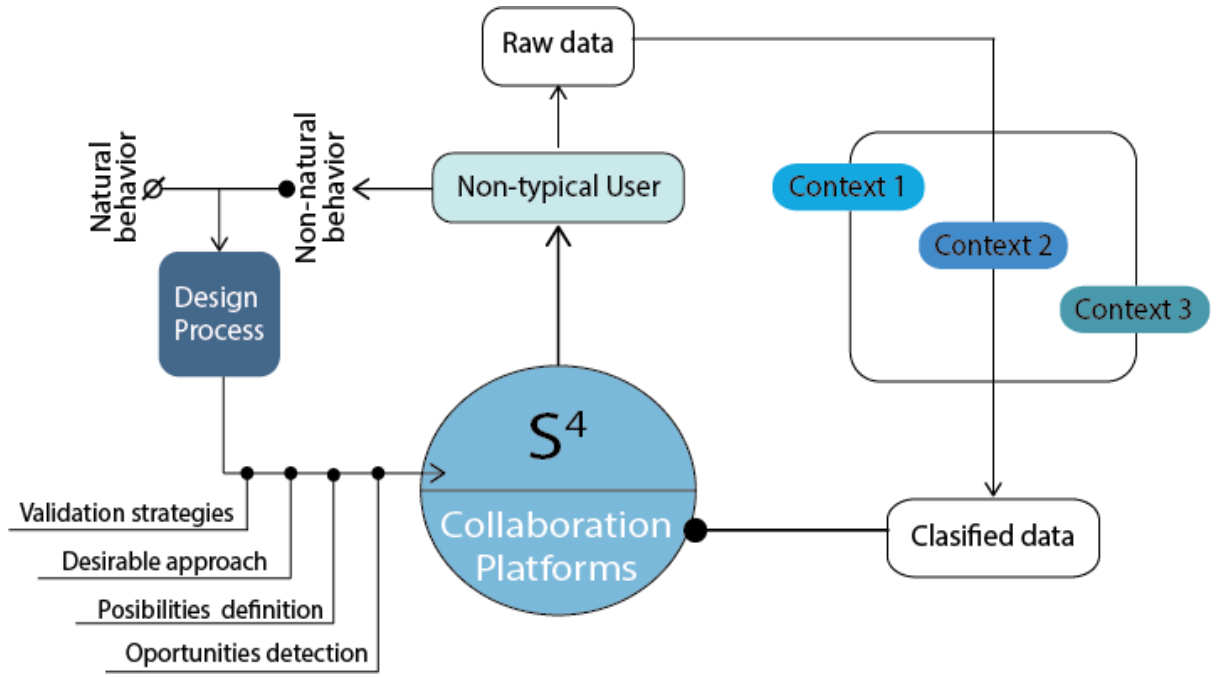

Fig. 3. Proposed learning framework.

This protocol includes three cycles of observing the possible challenging features of interactive devices, used by people with disabilities and people over 65 years old in participatory action research processes. Those cycles will be held with engineering and design bachelor students, using as a learning framework the integration explained in Fig. 3. Action research methodologies propose to use the following steps: planning, acting, observing, and reflecting, allow an understanding of the problem [22].

a) First Cycle: This will happen simultaneously in two separated contexts, one for industrial design students and one for engineering students. The purpose of doing so is to let students do their regular design process according to their mayor. Ideally, from those events valuable information about how to 
observe the outliers will emerge. That information will be useful in the second cycle to propose a validation tool, for both mayors: design and engineering.

In a design class, students must develop an assistive device for people with disabilities and the specific goal of recording the users' behaviour and actions. The desired outcome of this step is to observe the design process when pursuing including the outlier's behaviour and needs.

At the same time, in an engineering class, students will develop a systems interphase, to collect information from users' behaviour. This will allow to identify the most challenging aspects for non-typical users- in this case elders- when interacting with an interphase. Students should obtain information about the user's performance and its recording possibilities within system. In this context, the interphase should control a Sensing, Smart and Sustainable System. This means, that the product's features should include at least the following capabilities: monitoring, control, optimization and autonomy [1].

b) Second Cycle: This stage's goal will be to propose a validation tool that would allow to apply to products a systematic heuristic analysis (SHA) in a predictive way. Using the previous stage results, challenging interphase features will be used as analysing items. In this case, there will not be a proper interaction with the target users, instead there will be a Participatory Action Research with students. This process will allow to gather possible perceptions from a group of participants, to set a data base that would provide information for the SHA validation tool. Thus, the desired outcome for this cycle a preliminary system to set, in the next cycle, a systemic validation tool. This should be able to determine the most challenging features for a non-typical user when interacting with an S4 device interphase.

c) Third Cycle: The last moment of this research protocol should conclude with the description of the Design Methodology for inclusive Sensible, Smart, Sustainable and Social PSS. The pursued outcome is the possibility to reframe usability problems, to speed up innovation, by observing what has been ignored yet or labelled as an outlier.

To do so, a third action research cycle would take place in a design class to generate a design brief for inclusive S4 device as a PSS. As mentioned above, a design brief should contain a list of product/service requirements, achieved by the design team, concluding with a design proposal. The final research stage would use the previous cycle outcomes: the identified challenging features description and the systemic validation tool. From those elements, design students should search for the most convenient way of designing S4 PSS, while generating a device proposal (the specific type of device will be delineated after the First Cycle).

Furthermore, in the context of significant and experimental learning, this final research cycle sets an opportunity of presenting a challenge for the students: how to design in a global perspective for who has been considered as a minority. Understanding patterns, visualizing trends, predicting interactions and reframing the problem of attending non-articulated needs, ideally, would lead this research to alternatives that would facilitate the definition of a valid design methodology.

\section{Conclusion}

This paper shows a brief introduction to the previous research to establish the state of the art in the topic of collaboration platforms and the interaction types between devices, users and products and service developers. From the literature review, it is noticeable that there is an opportunity area for research in analysing the performance of non-typical users, along with the design considerations for Smart, Sensing, Sustainable and Social products and services systems.

The presented challenge is to demonstrate the applications in both disciplines, design and engineering, 
while providing a design tool, through validation of product features and recording the human input. This demonstration will take place through action research methodology to be developed in the next two years; however, different questions come up at early stages: Is it possible to use this framework for typical users? If so, which is the relevance of observing non-typical users? Is there a different way to include vulnerable population in the S4 perspective? Will the elder population in the future be a non-typical user?

The future actions of this work will focus on answering these interrogatives, combining collaboration research in Tecnologico de Monterrey University, through the Open Innovation Lab, along with a review of newest devices predictive performance around the world. The devices selection should be under criteria still to be determined and considering the validation strategy to identify the features more prone to cause confusion for the user.

\section{Conflict of Interest}

The authors declare no conflict of interest.

\section{Author Contributions}

Inés Alvarez-Icaza conducted the research and wrote the paper; Arturo Molina and Rogelio Bustamante-Bello advised the research; all authors had approved the final version.

\section{Acknowledgment}

The authors would like to acknowledge the financial support of Writing Lab, TecLabs, Tecnologico de Monterrey, Mexico, in the production of this work.

\section{References}

[1] Porter, M. E., \& Heppelmann, J. E. (2014). How smart, connected products are transforming competition. Harvard Business Review, 92(11), 64-88.

[2] Lilis, G., Conus, G., Asadi, N., \& Kayal, M. (2017). Towards the next generation of intelligent building: An assessment study of current automation and future IoT based systems with a proposal for transitional design. Sustainable Cities and Society, 28(55), 473-481.

[3] Molina, A. (2019). S3 Systems Theory. Springer.

[4] Mazzocchi, S. (2004). Open innovation: The new imperative for creating and profiting from technology. Innovation, 6(3), 474.

[5] Silva, M., Howells, J., \& Meyer, M. (2018). Innovation intermediaries and collaboration: Knowledgebased practices and internal value creation. Research Policy, 47(1), 70-87.

[6] Abbate, T., Luca, D., Gaeta, A., Lepore, M., Miranda, S., \& Perano, M. (2015). Analysis of open innovation intermediaries platforms by considering the smart service system perspective. Procedia Manufacturing 3, 3575-3582.

[7] Schuh, G., Potente, T., Wesch-Potente, C., Weber, A. R., \& Prote, J. P. (2014). Collaboration mechanisms to increase productivity in the context of industrie 4.0. Procedia Cirp, 19, 51-56.

[8] Arruda-Torres, P. M. (2018). An overview on strategic design for socio-technical innovation. Strategic Design Research Journal, 11(3), 186-192.

[9] Manzini, E. (2015). Design, When Everybody Designs: An Introduction to Design for Social Innovation. MIT Press.

[10] Nambisan, S. (2009). Platforms for collaboration. Stanford Social Innovation Review, 7(3), 44-49.

[11] Weichhart, G., Molina, A., Chen, D., Whitman, L. E., \& Vernadat, F. (2016). Challenges and current developments for sensing, smart and sustainable enterprise systems. Computers in Industry, 79, 34-46. 
[12] Herter, J., \& Ovtcharova, J. (2016). A model based visualization framework for cross discipline collaboration in Industry 4.0 scenarios. Procedia CIRP, 57, 398-403.

[13] Hermann, M., Pentek, T., \& Otto, B. (2016, January). Design principles for industrie 4.0 scenarios. Proceedings of the 2016 49th Hawaii International Conference on System Sciences (HICSS) (pp. 3928-3937).

[14] Romero, D., \& Molina, A. (2011). Collaborative networked organisations and customer communities: Value co-creation and co-innovation in the networking era. Production Planning \& Control, 22(5-6), 447-472.

[15] Camarinha-Matos, L. M., \& Afsarmanesh, H. (2005). Collaborative networks: A new scientific discipline. Journal of Intelligent Manufacturing, 16(4-5), 439-452.

[16] W. H. Organization. (2020). Ageing and Health. Retrieved from https://www.who.int/news-room/fact-sheets/detail/ageing-and-health

[17] Norton, L. (2018). Action Research in Teaching and Learning: A Practical Guide to Conducting Pedagogical Research in Universities. Routledge.

[18] Bennett, N. J., Blythe, J., Cisneros-Montemayor, A. M., Singh, G. G., \& Sumaila, U. R. (2019). Just transformations to sustainability. Sustainability, 11(14), 3881.

[19] McAteer, M. (2014). What is this thing called action research? Action Research in Education, 1-14.

[20] Kumar, V. (2012). 101 Design Methods: A Structured Approach for Driving Innovation in Your Organization. John Wiley \& Sons.

[21] Brown, T., \& Katz, B. (2019). Change by Design: How Design Thinking Transforms Organizations and Inspires Innovation (Vol. 20091). Harper Business.

[22] Kawamura, M. (2019). Perceived difficulties in group presentations. Action Research as an Intervention, $5(2)$.

Copyright (C) 2020 by the authors. This is an open access article distributed under the Creative Commons Attribution License which permits unrestricted use, distribution, and reproduction in any medium,

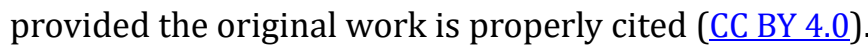

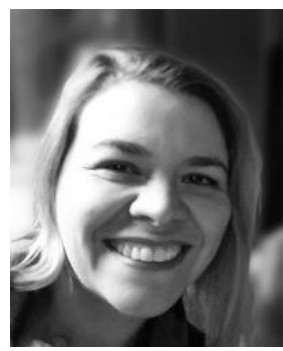

Inés Alvarez-Icaza Longoria was born in Mexico City, 1976. She is an industrial designer by the Universidad Nacional Autnoma de México in 2003. She obtained her master's in design, arts and science in the subject of design, education and technology by the Universidad Autónoma Metropolitana-Xochimilco in the year 2013. She has started her PhD in engineering in 2019 at Tecnológico de Monterrey, México City Campus, assessed by Dr Arturo Molina.

Now she is a full-time professor at Tecnológico de Monterrey since 2014, and the director of Industrial Design Bachelor Program from 2015 to 2018. Also, she is the leader of Post Urban Living Innovation Program of Chiba University at Japan and founding member of Interuniversity Design Workshop in Mexico City. She has developed several interior design projects for accessibility and universal design, since 2002, for both public and private sector.

Prof. Alvarez Icaza has been a speaker at several public and academic events in institutions such as UNESCO and the Educational Infrastructure Institute (INIFED) in Mexico. She is a member of the Technical Work Group for INIFED to generate the Mexican Standard for the Educational Furniture Design. In addition, she is a peer evaluator of the Mexican Council for the Design Programs Evaluation (COMAPROD), since 2016, and has been part of the jury for the Mexican Design Biennale, the National Design Prize and the Design Spotlight Prize of the World Design Organization. 


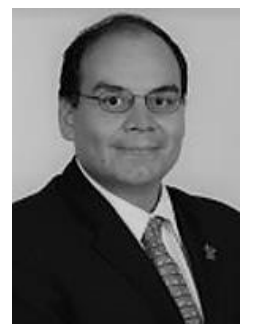

Arturo Molina was born in Oaxaca, Mexico, in 1964. He received the B.Eng. degree in computational systems and the M.Eng. degree in computational sciences from the Tecnológico de Monterrey, Monterrey, Mexico, the doctoral degree in mechanics from the Budapest University of Technology and Economics, Hungary, and the Ph.D. degree in manufacturing systems from the Loughborough University of Technology, UK.

Professor Molina is a member of the National Researchers System of Mexico (SNI-Nivel III), Mexican Academy of Sciences, among other relevant institutions. He had offer seminars and conferences in several countries and worked as a consultant for important corporations, such as World Bank, Inter-American Development Bank (IADB) and the United Nations Information and Communication Technologies (ICT) Task Force. He is the co-author of over 150 scientific papers in journals, conferences, chapter books, more than 4,000 citations (Google Scholar). His current research interests include concurrent engineering, technologies for rapid product design and manufacturing, enterprise integration engineering, technology management, and collaborative network organizations. He currently is the technical leader of the project: Bi-National Laboratory on Smart Sustainable Energy Management and Technology Training, funded by SENER - CONACYT.

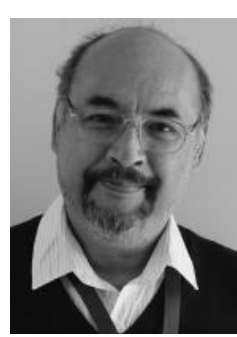

Rogelio Bustamante-Bello was born in Orizaba, Veracruz in 1965. He received the B.S. degree in electronic instrumentation from the Faculty of Physics and Mathematics of the Universidad Veracruzana, Xalapa, Ver; his master in electronic and telecommunications from CICESE, Ensenada, Baja California, México and his Ph.D. on telecommunications and electronics from SEPI, Instituto Politécnico Nacional. He has worked more than 12 years in advanced bioinstrumentation systems, applying them to the Driving Assistance Systems (ADAS), as well as the development of Healthy Active Spaces (Emotional Domotics) and exoskeletons attending the movement of lower limbs; bioinstrumentation systems, to file multiple patents with IMPI and WIPO. He is the director of the doctoral program in engineering studies at Tecnologico de Monterrey Institute in Mexico City Campus. 